\title{
Lower limb deep venous thrombosis due to vertebral osteophyte: a May-Thurner-like syndrome
}

\author{
Hasan Aydın๑, Mesut Engin $\odot$ \\ Department of Cardiovascular Surgery, University of Health Sciences, Mehmet Akif Inan Training and Research Hospital, Şanlurfa, \\ Turkey
}

\begin{abstract}
Lower extremity venous thrombosis is a risky condition that may cause crucial complications including pulmonary embolism and post-thrombotic syndrome. May-Thurner syndrome is a venous compression syndrome resulting in compression of the left common iliac vein by the overriding right iliac artery. In this case, left common iliac vein compression arises from the vertebral osteophyte.

Keywords: Vertebral osteophyte, May-Thurner syndrome, venous thrombosis, Cockett syndrome
\end{abstract}

M ay-Thurner Syndrome (MTS) was first observed in 1851 by Virchow as the anatomical variation of the left common iliac vein. However, the pathophysiological description of this disease was made by May-Thurner in 1956. This disease in which the left common iliac vein is exposed to external compression is also known as iliac venous compression syndrome, Iliocaval compression syndrome and Cockett syndrome [1]. Depending on this compression situation, the risk of lower extremity deep venous thrombosis (DVT) is increasing.

DVT is a serious condition that may lead to fatal complications like pulmonary embolism; therefore, it should be treated as soon as it is diagnosed. The etiology of DVT is explained by the Virchow triad. These are stasis in blood flow, vessel wall and coagulation disorders. The most serious complication of DVT which is almost the same frequency in both genders is the pulmonary embolism. Although the frequency of DVT is $1 / 1000$ in the general population, its frequency is increasing in the elderly, and immobile population
[2]. Early diagnosis of the disease and its treatment strategy are extremely important.

\section{CASE PRESENTATION}

A 61-year-old man was admitted to our emergency department with complaints of pain and swelling in the left lower extremity. Venous doppler ultrasonography revealed acute thrombosis in the popliteal and femoral veins. His medical history did not contain any risk factors like immobilization, malignancy or a systemic disease. Accordingly, we performed computed tomographic angiography (CTA) to exhibit the etiology of the venous thrombosis. CTA showed a vertebral osteophyte that caused compression of the left common iliac vein (Fig.1.AD). The patient was hospitalized and received low molecular weight heparin therapy with warfarin. He was discharged one week later when international normalized ratio (INR) was effective value (INR: 2.5- 
3).Patient did not have any complaints during 1-year follow-up and INR values were within normal range for the patients.

\section{DISCUSSION}

MTS is a venous compression syndrome resulting in compression of the left common iliac vein by the overriding right iliac artery. The causes of ilocaval compression syndrome include; iliac venous thrombosis, arterial compression-related web (MayThurner), previous DVT-related scar, pelvic cancer / abscess, fibroids / fibroids resulting with uterine hypertrophy, pregnancy, aorta-iliac aneurysms, postoperative hematome and vertebral osteophytes [3]. Doppler ultrasonography (DUSG) is the first option in diagnosis. The DUSG, which is used as the most common diagnostic tool, may cause the possible misdiagnosis of iliocaval compression when no further tests are performed. In clinical suspicious cases, CT angiography and venography should be used as a diagnostic method. In particular, the underlying iliocaval compression syndromes should be considered in repeated left lower extremity DVTs. That left lower extremity DVTs are 3-8 times higher than that of right side can be explained by compression syndromes. In a rare case of situs inversus totalis, MTS may be presented with the right lower extremity DVT clinic [4]. In the physical examination and clinical condition of the patient, this detail should not be overlooked.

The most common treatment for DVT is to start warfarin therapy with low molecular weight heparin and to provide effective INR range. The duration of treatment is six months for patients with the first DVT whose underlying causes are unknown. In patients with recurrent DVT, pulmonary embolism, or blood diseases, treatment may continue for life. New anticoagulant treatment options have been replaced in recent years [5]. The advantage of these treatment options is not requiring INR follow-up.

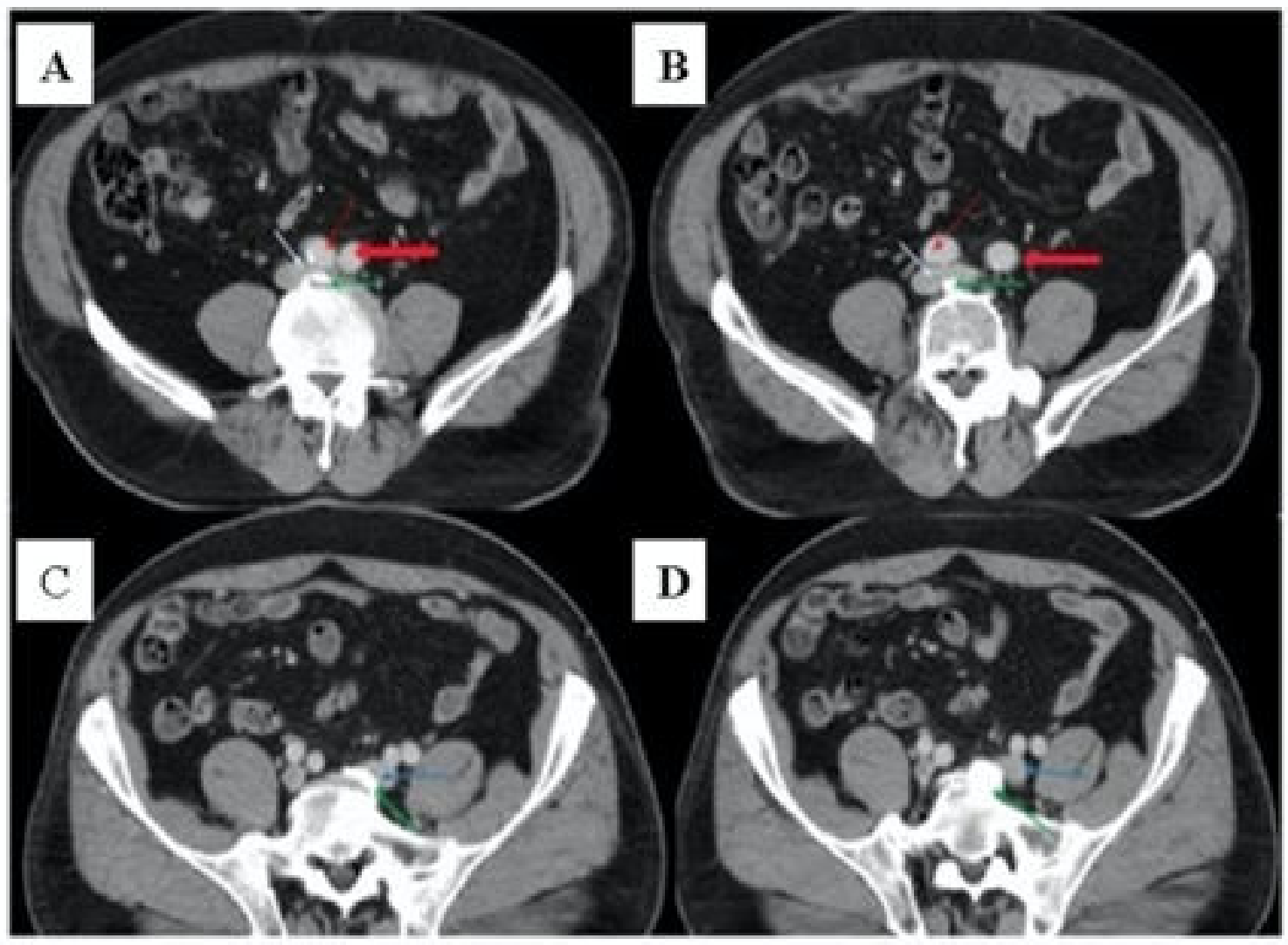

Fig. 1. (A, B, C, D) Computed tomographic angiographic images of compressing common iliac vein by the vertebral osteophyte (Yellow arrow: vertebral osteophte, Light blue arrow: vena cava inferior bifurcation, Blue arrow: left common iliac vein, Thick red arrow: left common iliac artery, Thin red arrow: right common iliac artery). 
Recently, endovascular treatment options such as mechanical thrombectomy have been included in addition to the medical treatment. That patients with MTS have symptoms is a requirement for treatment. Le et al. [6] had 111 MTS patients underwent endovascular treatment. Patients undergoing thrombectomy were treated with stents to eliminate pressure. After the operation, these patients were followed for six months with warfarin or rivaroxaban (Xarelto, Bayer Pharma AG, Berlin, Germany) [6]. However, patient with vertebral osteopathy wasn't observed in this large group of patients.

When we evaluated our case, the iliac compression of our patient was due to vertebral osteophytes which was a rare condition. For our patient, no endovascular treatment was planned against possible damage to the vessel due to osteophyte. In addition, his symptoms regressed under medical follow-up. Surgical treatment options such as venous bypass may be considered in patients with recurrent DVT and pulmonary embolism whose symptoms do not regress.

\section{CONCLUSION}

In conclusion, the treatment of the patient with vertebral osteophyte, which is a rare cause of ilioocaval compression syndrome, can be provided with medical treatment. Surgery can be considered in untreated patients.

\section{Informed consent}

Written informed consent was obtained from the patient for publication of this case report and any accompanying images.

\section{Conflict of interest}

The authors declared that there are no potential conflicts of interest with respect to the research, authorship, and/or publication of this article.

\section{Acknowledgement}

The article was presented as poster (EP-337) at the $15^{\text {th }}$ Congress of the Turkish Society of Cardiovascular Surgery, October 26-29, 2018, Antalya, Turkey.

\section{REFERENCES}

1. Gil Martín AR, Carreras Aja M, Arrieta Ardieta I, Labayen Azparren I. Cockett's syndrome, May-Thurner syndrome, or iliac vein compression syndrome. Radiologia 2014;56:e5-8.

2. Gong B, Xu Q, Pang Y, Hu J. Clinical features of patients with venous thromboembolism: 177 case analysis in 10 years. Zhonghua Wei Zhong Bing Ji Jiu Yi Xue 2019;31:453-7.

3. Birn J, Vedantham S. May-Thurner syndrome and other obstructiveiliac vein lesions: meaning, myth, and mystery. Vasc Med 2015;20:74-83.

4. Im S, Lim SH, Chun HJ, Ko YJ, Yang BW, Kim HW. Leg edema with deep venous thrombosis-like symptoms as an unusual complication of occult bladder distension and right MayThurner syndrome in a stroke patient: a case report. Arch Phys Med Rehabil 2009;90:886-90.

5. Agnelli G, Berkowitz S, Bounameaux H, Büller H, Cohen A, Gallus A, et al.; The EINSTEIN Investigators. Oral rivaroxaban for symptomatic venous thromboembolism. N Engl J Med 2010;363:2499-510.

6. Le TB, Lee TK, Park KM, Jeon YS, Hong KC, Cho SG. Contralateral deep vein thrombosis after iliac vein stent placement in patients with May-Thurner syndrome. J Vasc Interv Radiol 2018;29:774-80. 